Gut, 1984, 25, 151-157

\title{
Dermatitis herpetiformis: diagnosis, diet and demography
}

\author{
D J GAWKRODGER, J N BLACKWELL, H M GILMOUR, E A RIFKIND, \\ R C HEADING, AND R StC BARNETSON
}

Departments of Dermatology, Therapeutics, and Pathology, Royal Infirmary, Edinburgh, and Gastrointestinal Unit, Western General Hospital, Edinburgh

SUMmaRY We describe a long term study of 76 patients with dermatitis herpetiformis. Unlike patients with coeliac disease, where the peak incidence was during the first and fourth decades, no dermatitis herpetiformis patients presented in the first decade; also, there was a male preponderance in dermatitis herpetiformis which contrasts with the excess of females in coeliac disease. The apparent prevalence of dermatitis herpetiformis was 11 per 100000 in our population; approximately one fifth of that of coeliac disease. Jejunal villous atrophy was present in $78 \%$ of our dermatitis herpetiformis patients, and a single jejunal biopsy was as effective at detecting this as the multiple biopsy technique. A majority of patients were able to stop, or radically reduce their dapsone or sulphapyridine treatment after the institution of a gluten free diet. Spontaneous remission of the skin lesion occurred in only two patients not receiving a gluten free diet. Gastric parietal or thyroid antibodies were detected in $38 \%$ of patients, and three cases of thyroid disease and two cases of pernicious anaemia were detected. Lymphoma developed in two patients, one being intestinal in origin. We conclude that a gluten free diet is of therapeutic benefit in dermatitis herpetiformis and that spontaneous remission is uncommon in those not on a diet. Despite patchiness of the enteropathy, a single jejunal biopsy is quite adequate to diagnose the presence of upper intestinal villous atrophy.

Since the first report of the association of dermatitis herpetiformis with coeliac disease in $1966,{ }^{1}$ evidence of close links between the two disorders has accumulated. Well documented points of similarity include the response of the enteropathy to a gluten free diet, ${ }^{23}$ the high prevalence of HLA B8 and DR3 phenotypes, ${ }^{4-6}$ the occurrence of hyposplenism ${ }^{78}$ and a predisposition to the development of lymphoma. ${ }^{910}$ While these associations are not in dispute, controversy has arisen in relation to the major differences between the two disorders. By definition, the appearance of the skin lesions characterises dermatitis herpetiformis, but, while some authors believe that exclusion of gluten from the diet leads to remission of the skin disease,$^{11-14}$ others disagree. ${ }^{15}{ }^{16}$ Similarly, there is disagreement about the frequency with which intestinal villous

Address for correspondence: Dr R C Heading, Department of Therapeutics, Royal Infirmary, Edinburgh EH3 9YW.

Received for publication 14 April 1983 atrophy occurs in dermatitis herpetiformis. Some investigators have observed a normal jejunal mucosa in about one third of all patients with dermatitis herpetiformis, ${ }^{17} 18$ but Brow and coworkers ${ }^{19}$ have claimed that enteropathy is almost always demonstrable, provided a sufficient number of biopsies is taken. Evidence of patchiness of the intestinal lesion ${ }^{1920}$ has helped to create the suspicion that a single jejunal biopsy may not be an adequate basis for assessment of the small intestine in dermatitis herpetiformis, and that sampling variation may sometimes produce a normal biopsy from an abnormal intestine.

This report outlines our experience with dermatitis herpetiformis, which we believe is now large enough for us to comment on several current controversies, including the effect of a gluten free diet on the skin rash, the need for multiple small bowel biopsies, and the relationship of dermatitis herpetiformis with coeliac disease, and with recognised autoimmune disorders. 
Methods

\section{PATIENTS}

Seventy six patients with dermatitis herpetiformis, referred by consultants within the south east region of Scotland, have been investigated and followed up at a clinic run jointly by a dermatologist and a gastroenterologist over the last 11 years. The diagnosis of dermatitis herpetiformis required the demonstration by direct immunofluorescence of granular IgA deposits in the dermal papillae of uninvolved skin. If this test was negative or not performed a diagnosis of dermatitis herpetiformis was still tenable provided all of the following criteria were satisfied: (1) A pruritic symmetrical papular or vesicular eruption predominantly on extensor surfaces of limbs, shoulders and buttocks. (2) Improvement on treatment with dapsone or sulphapyridine. (3) Histopathological appearance of a subepidermal blister with an inflammatory infiltrate comprising predominantly neutrophils and eosinophils. (4) Subtotal or partial villous atrophy on small bowel biopsy.

These criteria were fulfilled in two patients, one having negative immunofluorescence on two skin biopsies and one whose skin had never been studied by immunofluorescence.

Small bowel biopsy was carried out in all patients except eight in whom it was contraindicated or refused. Single biopsies were performed using a Crosby or Watson capsule, sampling from the jejunum at the level of the ligament of Treitz. Multiple biopsies were obtained with a Quinton hydraulic suction biopsy instrument, taking six biopsies from the proximal jejunum back to the fourth part of the duodenum. A gluten free diet in which wheat, barley, and oat products were excluded, was started in all 53 patients who had villous atrophy, in five with normal small bowel biopsies who responded poorly to drug treatment and in three who had not had intestinal biopsies performed. Before the diet, the minimum dose of sulphapyridine or dapsone required to control the skin symptoms was established and after the diet had begun the patients were encouraged to reduce the dosage of drugs whenever possible and ultimately to stop them. Patients were reviewed three months after starting the diet and then usually at six monthly intervals.

Information on coeliac disease patients was obtained from the coeliac register compiled by the Gastrointestinal Unit of the Western General Hospital, Edinburgh. Data for sex and age at presentation of 324 coeliac patients in the Lothian Region born before 1963 were analysed together with figures for the prevalence of coeliac disease in
Lothian Region. ${ }^{21}$ Information on sex and age at presentation was obtained for 76 dermatitis herpetiformis patients from south-east Scotland and the prevalence of dermatitis herpetiformis in Lothian Region was calculated for comparison with the data for coeliac disease.

Where statistical significance is quoted, the method of analysis is the $\chi^{2}$ test.

\section{Results}

SEX AND AGE

The dermatitis herpetiformis patients comprised 49 men (64\%) and 27 women (36\%); a male to female ratio of $1 \cdot 81: 1$. The mean age at presentation was 43 years for men (range 16-75 years) and 38 years for women (range 12-77 years). The greatest number of patients presented in the fourth decade and there were no cases in the first 10 years of life (Fig. 1). This contrasts with coeliac disease which has its highest incidence during the first decade. If these early cases are excluded, however, the age distribution of coeliac disease is similar to dermatitis herpetiformis (Fig. 2). The coeliac disease patients showed a male to female ratio of $1: 1.89$ which is statistically different from that of dermatitis herpetiformis $(\mathrm{p}<0 \cdot 01)$.

The 15 dermatitis herpetiformis patients with normal jejunal biopsies were compared with the 53 who had villous atrophy. There were 12 men and

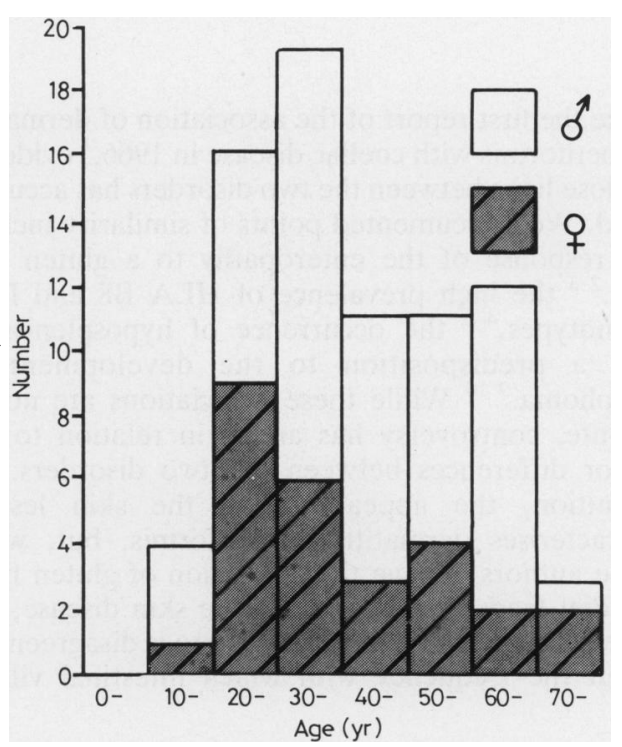

Fig. 1 Seventy six dermatitis herpetiformis patients: sex and age at presentation. 


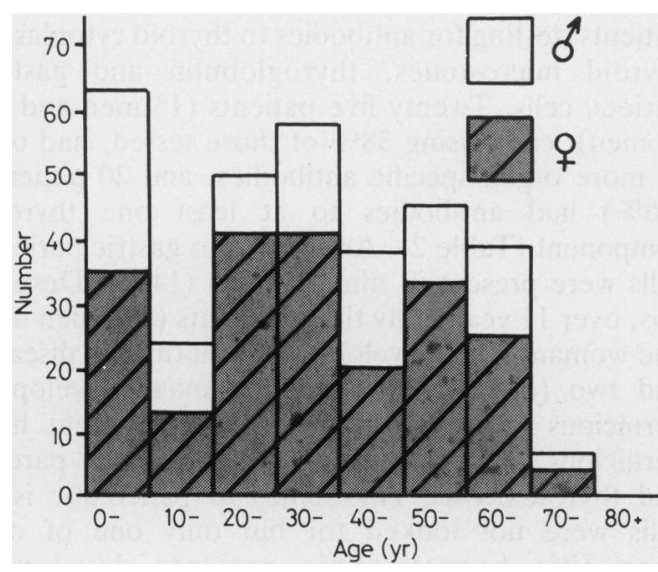

Fig. 2 Three hundred and twenty four coeliac disease patients: sex and age at presentation.

three women among the normal biopsy patients giving a male to female ratio of $4: 1$, which is higher than the 1.52:1 ratio found in the villous atrophy group although this difference is not statistically significant. Dermatitis herpetiformis cases with normal jejunal biopsies had a mean age at presentation of 52 years for men and 42 years for women, as compared with the slightly earlier presentation of the villous atrophy group at 43 years and 34 years respectively.

\section{PREVALENCE}

Analysis of prevalence of dermatitis herpetiformis and coeliac disease per 100000 living population for 10 year groups by year of birth in Lothian Region from 1900 revealed no overall correlation between the two sets of data. The overall prevalence of dermatitis herpetiformis in December 1981 was 11.5 per 100000 for the Lothian Region compared with the figure for coeliac disease of 56 per 100000 population also for Lothian Region (December 1979). For patients born between 1900 and 1959, the prevalence of coeliac disease was approximately five times that of dermatitis herpetiformis. No cases of dermatitis herpetiformis born after 1960 have yet been seen.

INCIDENCE OF JEJUNAL VILLOUS ATROPHY COMPARING SINGLE BIOPSY WITH MULTIPLE BIOPSY TECHNIQUES

Subtotal or partial villous atrophy was found in 53 $(78 \%)$ out of the 68 patients biopsied (Table 1). The 15 patients who had no villous atrophy all had normal villous heights, surface cell heights and intraepithelial lymphocyte counts, with the
Table 1 Result of jejunal biopsy

\begin{tabular}{llll}
\hline & \multicolumn{3}{l}{ Type of biopsy } \\
\cline { 2 - 4 } Histological appearance & Single & Multiple & All cases \\
\hline Partial/subtotal villous atrophy & 34 & 19 & 53 \\
Normal & 7 & 8 & 15 \\
All cases & 41 & 27 & 68 \\
\hline
\end{tabular}

exception of one, in whom the intraepithelial lymphocyte count was raised. The single biopsy method was used in 41 patients and $34(83 \%)$ of these had an abnormal jejunal mucosa. Multiple biopsies were performed in 27 patients, proving abnormal in $19(70 \%)$. There is no statistical difference between these results. Six of those studied by the multiple method showed some variation in degree of villous atrophy between biopsies and in two patients normal villi were identified in one third of the specimens with the remainder of the biopsies showing partial villous atrophy.

Although only three $(12 \%)$ out of 24 women biopsied had a normal jejunal mucosa, as compared with $12(27 \%)$ of 44 men, this difference does not reach statistical significance.

\section{EFFECT OF GLUTEN FREE DIET ON DRUG THERAPY}

Sixty one patients started a gluten free diet but 10 did not adhere to it and were asked to stop. Nine others admitted to occasional dietary indiscretion but the remaining 42 reported that they were strictly adhering to the diet. Of the 51 who continued on a gluten free diet, 27 patients (53\%) were able to stop dapsone or sulphapyridine completely and in 12 others $(23.5 \%)$ the drug requirements were reduced by at least $50 \%$. Twelve patients were not able to reduce their drug dosage but four of these have only been on the diet for less than 12 months.

Drug requirements fell to $50 \%$ of the pre-gluten free diet level in an average of 18 months after starting the diet for the 39 patients who managed to reduce their drug intake or subsequently discontinue it altogether (Fig. 3). The time to achieve this reduction ranged from one to 103 months with 17 patients taking less than six months. Twenty seven patients were able to discontinue their dapsone or sulphapyridine; the mean time required was 25 months after the gluten free diet began (range 1-91 months) and five patients stopped their medication in less than six months (Fig. 4). These 27 patients have remained off their dapsone or sulphapyridine for periods between three and 133 months (mean 49 months).

Three of the five patients with normal jejunal biopsies did not adhere to the gluten free diet and 


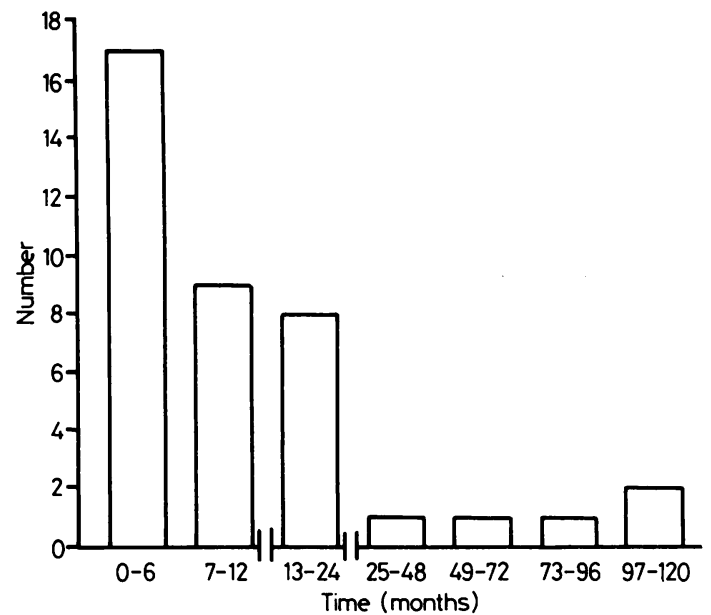

Fig. 3 Time after starting gluten free diet to halve drug dosage.

discontinued it. One of the remaining two patients has reduced the dose of dapsone by more than $50 \%$ but not stopped it, and the other has noticed no change in drug requirements.

Spontaneous remission of the rash of dermatitis herpetiformis has occurred in only two of the 25 patients who were not on, or did not continue on, a gluten free diet. This number of spontaneous remissions shows a statistically significant difference from the number of patients who, continuing on a gluten free diet, have been able to discontinue their drugs $(p<0.001)$. One of the patients who had a spontaneous remission had had a normal jejunal biopsy, but in the other, jejunal biopsy failed and further biopsy was refused.

PREVALENCE OF AUTOANTIBODIES AND AUTOIMMUNE DISEASE

Autoantibody screens were performed in 66

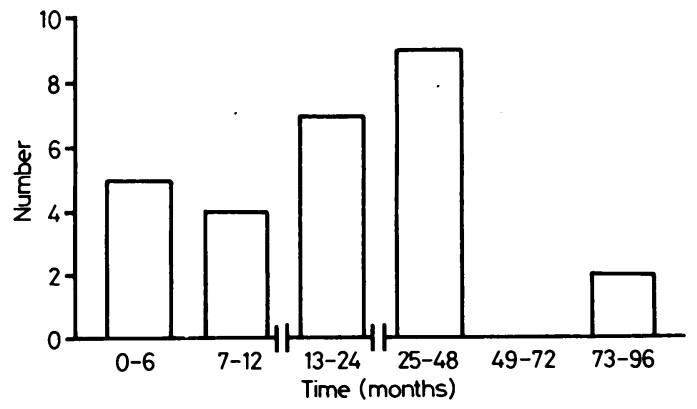

Fig. 4 Time after starting gluten free diet to stop drugs. patients, testing for antibodies to thyroid cytoplasm, thyroid microsomes, thyroglobulin and gastric parietal cells. Twenty five patients (15 men and 10 women), comprising $38 \%$ of those tested, had one or more organ specific antibodies, and 20 patients $(30 \%)$ had antibodies to at least one thyroid component (Table 2). Antibodies to gastric parietal cells were present in nine patients $(14 \%)$. Despite this, over 11 years only three patients (two men and one woman) have developed clinical thyroid disease and two (one woman and one man) developed pernicious anaemia. In two others a parent had pernicious anaemia and a further patient's parent had thyrotoxicosis. Antibodies to pancreatic islet cells were not looked for but only one of our dermatitis herpetiformis patients developed diabetes mellitus.

\section{Discussion}

The jejunal villous atrophy of dermatitis herpetiformis is known to improve with a gluten free diet, but there has been some disagreement about the skin response. ${ }^{15} 16$ Most recent authors report that the dose of dapsone or sulphapyridine can be reduced or stopped on gluten withdrawal without a recurrence of the rash. Our series confirms these findings with $53 \%$ of the patients who continued on a gluten free diet able to stop their drugs and a further $23.5 \%$ able to reduce the drug dosage significantly. In reports describing a poor skin response to a gluten free diet, ${ }^{315}$ only one patient was followed for more than nine months and this may explain the discrepancy. The villous atrophy of dermatitis herpetiformis patients improves within six months, ${ }^{3}$ and it remains a mystery why it often takes over two years for the skin lesions to disappear. Only two of our 25 patients not on a gluten free diet were able to discontinue their drugs without recurrence of the rash, suggesting that spontaneous remission is uncommon.

A gluten free diet allows the reduction or cessation of drugs which may have serious side effects, but are there any other reasons for its use? Two of

Table 2 Prevalence of autoantibodies (66 patients)

\begin{tabular}{lcc}
\hline Autoantibody & Number & Percentage \\
\hline Any organ specific & 25 & 38 \\
Any thyroid & 20 & 30 \\
Thyroid cytoplasm & 4 & 6 \\
Thyroid microsome & 15 & 23 \\
Thyroglobulin & 8 & 12 \\
All 3 thyroid & 2 & 3 \\
Gastric parietal cell & 9 & 14 \\
\hline
\end{tabular}


our patients, both with jejunal villous atrophy, have developed a lymphoma. The first died from a small bowel lymphoma despite chemotherapy, and the second, with a non-intestinal lymphoma is undergoing chemotherapy. Both had been on a gluten free diet although each admitted to some dietary indiscretion. A gluten free diet has not been shown to decrease the incidence of intestinal lymphoma in coeliac disease, ${ }^{922}$ and thus in dermatitis herpetiformis patients with villous atrophy, who rarely have malabsorption, the theoretical benefit of prescribing a gluten free diet purely for the enteropathy is questionable. Despite this lack of evidence, however, the current consensus favours such treatment, mainly because most patients are able to stop or reduce their drug intake by taking a gluten free diet.

Between a third and a fifth of dermatitis herpetiformis patients do not have small bowel villous atrophy, ${ }^{17} 18$ and it is puzzling that the skin lesions in a number of these respond to a gluten free diet. ${ }^{12}$ Brow et $a l^{19}$ believed the intestinal lesion to be patchy and concluded that mucosal abnormalities were almost invariably to be found if enough biopsies were taken. Only one of their 22 patients had a normal jejunal biopsy although six showed a histological variation from almost normal to completely flat. Scott and Losowsky ${ }^{20}$ found patchiness of villous atrophy in dermatitis herpetiformis and coeliac disease patients studied by multiple biopsy, and concluded that this investigation was 'invaluable'. Our findings confirm reports that the degree of villous atrophy is variable in some patients. Although two out of 27 of our dermatitis herpetiformis patients studied by multiple biopsy showed normal villi in one third of specimens, it is unlikely that a single biopsy would have been completely normal because it provides a larger sample of mucosa than that obtained each time with the multiple technique. Multiple biopsy is without doubt a useful research investigation but we have found no evidence that a single biopsy taken with a Watson or Crosby capsule is manifestly inferior to the multiple technique for diagnostic purposes.

Dermatitis herpetiformis and coeliac disease both have a higher prevalence of HLA B8 and DR3 phenotypes than the normal population. ${ }^{4-6}$ These HLA antigens are often associated with autoimmune disease, although neither dermatitis herpetiformis nor coeliac disease belongs in this category. This may, however, explain the increased prevalence of thyroid and parietal cell antibodies in our patients and in previous series. ${ }^{18} 23$ Achlorhydria or hypochlorhydria is present in over $50 \%$ of dermatitis herpetiformis cases, ${ }^{24}$ and suggests that pernicious anaemia might be common in dermatitis herpetiformis. Despite the raised prevalence of autoantibodies in our group, autoimmune thyroid disease and pernicious anaemia were encountered relatively infrequently.

Granular IgA deposits in the dermal papillae of non-involved skin are said by some to be mandatory for diagnosing dermatitis herpetiformis. ${ }^{25}$ Patients have been described with negative immunofluorescence, however, but with all the clinical hallmarks of this disease. ${ }^{1326} \mathrm{We}$ are not convinced that the demonstration of granular $\operatorname{IgA}$ is an absolute prerequisite provided all other criteria are satisfied, and we have included any patients able to meet these.

Coeliac disease results from an abnormal response of the small bowel to gluten and is associated with altered immunological function. ${ }^{27} \mathrm{~A}$ similar situation may exist in dermatitis herpetiformis, although the pathogenetic mechanisms here must in addition explain the skin lesions. We find that dermatitis herpetiformis is nearly twice as common in men as in women; this confirms previous observations, ${ }^{28}{ }^{29}$ and is almost the reverse of the finding in coeliac disease. The frequent presentation of coeliac disease in the first decade contrasts with the rarity of such an early occurrence in dermatitis herpetiformis, although thereafter the age incidences are similar. We are unable to show any correlation between the prevalence of coeliac disease and dermatitis herpetiformis by year of birth, a finding which, if positive, might have suggested an environmental factor influencing both disorders. Between 1975 and 1979, there was a two fold increase in the number of coeliacs diagnosed in the Lothian Region compared with the preceding five years. ${ }^{30}$ No corresponding increase in the number of dermatitis herpetiformis patients has been noted.

The exact relationship between the skin and gut lesions of dermatitis herpetiformis remains obscure, although the fact that both respond to a gluten free diet suggests that they are linked and that gluten may be involved in the pathogenesis of the rash. The $\operatorname{IgA}$ in the skin has characteristics of dimeric $\operatorname{IgA}$ and hence probably originates in the gut. ${ }^{31} \operatorname{IgA}$ containing circulating immune complexes were found to be significantly raised after the oral ingestion of wheat in five out of five dermatitis herpetiformis patients, two with previously normal IgA circulating immune complex levels. ${ }^{32}$ This suggests that IgA circulating immune complexes are stimulated by ingested wheat antigens (presumably gluten) and then lodge in the dermal papillae for as yet unknown reasons. Circumstantial evidence for a circulating factor comes from our observation of one patient whose dapsone requirement fell for six 
months after a major gastrointestinal haemorrhage which complicated jejunal biopsy and necessitated a transfusion of 15 units of blood. The rash of dermatitis herpetiformis, however, is unlikely to be due simply to wheat antigen induced circulating immune complexes because an average of two years on a gluten free diet is required for the eruption to clear and dermal IgA persists long after the rash has cleared on gluten exclusion. ${ }^{33}$

Our findings confirm the beneficial effect of a gluten free diet and a single jejunal biopsy can now be used with confidence to detect intestinal villous atrophy in dermatitis herpetiformis. We have highlighted areas of disparity as well as points of similarity between dermatitis herpetiformis and coeliac disease, but the relationships between these disorders, and between gluten and the rash, remain unexplained despite recent immunological advances. Future research may resolve these difficulties by concentrating on the differences between dermatitis herpetiformis and coeliac disease, rather than pursuing points of similarity.

We thank Dr W J Irvine whose laboratory performed the thyroid and gastric parietal cell antibody assays, and are grateful to those consultant dermatologists who have referred cases to us. We thank Dr W D H Conacher and Dr M K Cook for allowing us to quote details of the second patient with lymphoma. This work was supported in part by a grant from the Scottish Home and Health Department.

\section{References}

1 Marks J, Shuster S, Watson AJ. Small bowel changes in dermatitis herpetiformis. Lancet 1966; 2: 1280-2.

2 Fry L, McMinn RMH, Cowan JD, Hoffbrand AV. Gluten free diet and reintroduction of gluten in dermatitis herpetiformis. Arch Dermatol 1969; 100: 129-35.

3 Weinstein WM, Brow JR, Parker F, Rubin CE. The small intestinal mucosa in dermatitis herpetiformis II. Relationship of the small intestinal lesion to gluten. Gastroenterology 1971; 60: 362-9.

4 Katz SI, Falchuk ZM, Dahl MV, Rogentine GN, Strober W. HL-A8: a genetic link between dermatitis herpetiformis and gluten sensitive enteropathy. J Clin Invest 1972; 52: 2977-80.

5 White AG, Barnetson RStC, Da Costa JAG, McClelland DBL. The incidence of HL-A antigens in dermatitis herpetiformis. Br J Dermatol 1973; 89: 133-6.
6 Keuning JJ, Pena AS, van Leeuwen A, van Hoof JP, van Rood JJ. HLA-DW3 associated with coeliac disease. Lancet 1976; 1: 506-8.

7 Pettit JE, Hoffbrand AV, Seah PP, Fry L. Splenic atrophy in dermatitis herpetiformis. Br Med J 1972; 2: 438-40.

8 McCarthy CF, Fraser ID, Evans KT, Read AE. Lymphoreticular dysfunction in idiopathic steatorrhoea. Gut 1966; 7: 140-8.

9 Harris OD, Cooke WT, Thompson H, Waterhouse JAH. Malignancy in adult coeliac disease and idiopathic steatorrhoea. Am J Med 1967; 42: 899-912.

10 Reunala $T$, Helin $H$, Kuokkanen $K$, Hakala $T$. Lymphoma in dermatitis herpetiformis: report on four cases. Acta Derm Venereol (Stockh) 1982; 62: 343-6.

11 Heading RC, Paterson WD, McClelland DBL, Barnetson RStC, Murray MSW. Clinical response of dermatitis herpetiformis skin lesions to a gluten free diet. $\mathrm{Br} J$ Dermatol. 1976; 94: 509-14.

12 Reunala T, Blomqvist K, Tarpila S, Halme H, Kangas $\mathrm{K}$. Gluten free diet in dermatitis herpetiformis. 1. Clinical response of skin lesions in 81 patients. $\mathrm{Br} J$ Dermatol 1977; 97: 473-80.

13 Frodin T, Gotthard R, Hed J, Molin L, Norrby K, Walan A. Gluten free diet for dermatitis herpetiformis: the long term effect on cutaneous immunological and jejunal manifestations. Acta Derm Venereol (Stockh) 1981; 61: 405-11.

14 Fry L, Leonard JN, Swain F, Tucker WFG, Haffenden G, Ring N, McMinn RMH. Longterm follow-up of dermatitis herpetiformis with and without dietary gluten withdrawal. Br J Dermatol 1982; 107: 631-40.

15 Shuster S, Watson AJ, Marks J. Coeliac syndrome in dermatitis herpetiformis. Lancet 1968; 1: 1101-6.

16 Shuster S. Dermatology in internal medicine. Oxford: Oxford University Press, 1978: 133.

17 Fry L, Keir P, McMinn RMH, Cowan JD, Hoffbrand AV. Small intestinal structure and function and haematological changes in dermatitis herpetiformis. Lancet 1967; 2: 729-34.

18 Davies MG, Marks R, Nuki G. Dermatitis herpetiformis - a skin manifestation of a generalised disturbance in immunity. $Q J$ Med 1978: 186: 221-48.

19 Brow JR, Parker F, Weinstein WM, Rubin CE. The small intestinal mucosa in dermatitis herpetiformis I: severity and distribution of the small intestinal lesion and associated malabsorption. Gastroenterology 1971; 66: $355-61$.

20 Scott BB, Losowsky MS. Patchiness and duodenaljejunal variation of the mucosal abnormality in coeliac disease and dermatitis herpetiformis. Gut 1976; 17: 984-92.

21 Rifkind EA, Logan RFA, Busuttil A, Gilmour H, Ferguson A. Coeliac disease in Edinburgh and the Lothians 1900-1980. Scot Med J 1982; 27: 256.

22 Holmes GKT, Stokes PL, Sorahan TM, Prior P, Waterhouse JAH, Cooke WT. Coeliac disease, gluten free diet and malignancy. Gut 1976; 17: 612-9.

23 Fraser NG. Autoantibodies in dermatitis herpetiformis. Br J Dermatol 1970; 83: 609-13.

24 Heading RC, Parkin DM, Barnetson RStC, McClelland DBL, Shearman DJC. Small intestinal bacterial 
flora in dermatitis herpetiformis. Am J Dig Dis 1974; 19: 704-8.

25 Fry L, Seah PP. Dermatitis herpetiformis: an evaluation of diagnostic criteria. Br J Dermatol 1974; 90: $137-46$.

26 Cooper BT, Mallas E, Trotter MD, Cooke WT. Response of the skin in dermatitis herpetiformis to a gluten free diet with reference to jejunal morphology. Gut 1978; 19: 754-8.

27 Asquith P, Haeney MR. Coeliac disease. In: Asquith P ed. Immunology of the gastrointestinal tract. Edinburgh: Churchill Livingstone, 1979: 66-94.

28 Eyster WH, Kierland RR. Prognosis of dermatitis herpetiformis treated and untreated. Arch Dermatol 1957: 64: 1-8.

29 Alexander JO'D. Dermatitis herpetiformis. London:
WB Saunders, 1975; 30.

30 Logan RFA, Tucker G, Rifkind EA, Heading RC, Ferguson A. Changes in the clinical features of adult coeliac disease in Edinburgh and the Lothians 19661979. Br Med J 1983; 286: 95-7.

31 Unsworth DJ, Payne AW, Leonard JN, Fry L, Holborow EJ. IgA in dermatitis herpetiformis is dimeric Lancet 1982; 1: 478-80.

32 Zone JJ, La Salle BA, Provost TT. Induction of IgA circulating immune complexes after wheat feeding in dermatitis herpetiformis patients. J Invest Dermatol 1982; 78: 375-80.

33 Fry L, Haffenden G, Wojnarowska FT, Thompson BR, Seah PP. IgA and C3 complement in the uninvolved skin in dermatitis herpetiformis after gluten withdrawal. Br J Dermatol 1978; 99: 31-7. 